\title{
AERATION EFFECT ON SPIRULINA PLATENSIS GROWTH AND $\gamma$-LINOLENIC ACID PRODUCTION
}

\author{
Srinivasa Reddy Ronda ${ }^{*}$,Chandra Sekhar Bokka, Chandrika Ketineni, Binod Rijal, Prasada Rao Allu
}

Department of Biotechnology, K L University, Vaddeswaram-522502, Guntur, India.

Submitted: October 29, 2009; Returned to authors for corrections: April 15, 2011; Approved: January 16, 2012.

\begin{abstract}
The influence of aeration on algal growth and gamma-linolenic acid (GLA) production in a bubble column photobioreactor was investigated. Studies were performed in a $20-\mathrm{L}$ reactor at different aeration rates $(0.2-$ $2.5 \mathrm{vvm}$ ). Static, continuous, and periodic operation of air resulted in $41.9 \%, 88.4 \%$, and $108 \%$ air saturation of dissolved oxygen, for which the corresponding values of GLA were $2.3,6.5$, and $7.5 \mathrm{mg} \cdot \mathrm{g}^{-1} \mathrm{dry}$ cell weight, respectively. An increase in the aeration rate from 0.2 to $2.5 \mathrm{vvm}$ enhanced both the specific growth rate and GLA content under periodic sparging in the bicarbonate medium. With a 6-fold increase in the aeration rate, the GLA content of the alga increased by $69.64 \%$ (5.6-9.5 $\mathrm{mg} \cdot \mathrm{g}^{-1}$ dry cell weight). In addition, the total fatty acid (TFA) content in dry biomass increased from $2.22 \%$ to $4.41 \%$, whereas the algae maintained a constant GLA to TFA ratio within the aeration rate tested. The dependence of GLA production on the aeration rate was explained by interrelating the GLA production rate with the specific growth rate using the Luedeking and Piret mixed growth model.
\end{abstract}

Key words: Spirulina platensis; Aeration; Photobioreactor; Gamma-linolenic acid

\section{INTRODUCTION}

High-value metabolite production from diverse algal sources is a subject of interest in algal biotechnology $(3,4)$. Gamma-linolenic acid (GLA) is an important polyunsaturated fatty acid (PUFA) with wide applications in treating various disorders such as rheumatoid arthritis (22), eczema (21), diabetes, multiple trauma, and premenstrual syndrome (5). The potential benefits of using PUFAs in various medical formulations were reported (date back to 1975) when the Food and Agricultural Organization and World Health Organization recommended the inclusion of these fatty acids including GLA in infant formula, as the human body lacks the key enzymes involved in the synthesis of PUFAs. Although plants are the traditional sources for GLA production, novel alternative sources among microbes include fungi and microalgae. Among microalgae, Spirulina platensis is known to accumulate large quantities of GLA (31\% of total fatty acids (TFAs) and 1.0$1.4 \%$ dry cell wt.) (16). In addition, algae are potential sources of many high-value metabolites such as proteins, natural pigments, and antioxidants (8). GLA is an essential fatty acid found in the membrane-bound lipids (galactolipids) of the alga. A series of membrane-bound proteins, viz. NADPHcytochrome $\mathrm{b}_{5}$ reductase, cytochrome $\mathrm{b}_{5}$, and a terminal

*Corresponding Author. Mailing address: Department of Biotechnology, K L University, Greenfields, Vaddeswaram-522502, Guntur district, Andhra Pradesh, India.; E-mail: srinus4@rediffmail.com 
desaturase, are involved in the synthesis of unsaturated fatty acids (6). In addition, oxygen is also required for unsaturated fatty acid synthesis (1). In algae, the desaturation of linoleic acid (C18:2 $\omega 6)$ is facilitated by either $\Delta-6$ desaturase or $\Delta-15$ desaturase, resulting in the production of $18: 3 \omega 6$ or $18: 3 \omega 3$, respectively; these fatty acids ultimately form higher PUFAs,

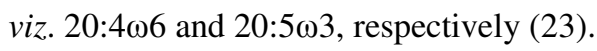

Commercially, cyanobacteria are grown in open ponds; however, the operating parameters in such cultivation ponds are not controlled, and hence, many studies have exploited the option of using an enclosed photobioreactor. It has the advantage of better control over the operating conditions such as the aeration rate, light intensity, temperature, and $\mathrm{pH}$. Different enclosed photobioreactor designs employed for algal cultivation include the airlift reactor, bubble column (17), gasinduced mechanically agitated reactor (10), tubular photobioreactor (18), and flat panel reactor (19).Pneumatically agitated column photobioreactors (airlift, bubble column, and tubular) are preferred over stirred vessels because of their low power consumption, better light availability per unit area, and low shear. The only disadvantage of pneumatically agitated photobioreactors is their lower mixing efficiency. Furthermore, continuous aeration in bicarbonate-enriched medium in such reactors is known to result in great losses of total inorganic carbon in the form of $\mathrm{CO}_{2}$ in alkaline conditions $(2,15)$. Additionally, it results in saturation levels of dissolved oxygen (13). The present work was undertaken to investigate the effects of aeration on microalgal growth and GLA production. GLA was selected because its synthesis requires oxygen, and it would be interesting to study the effect of aeration on the production of the metabolite. The work also establishes a relationship between GLA production and the aeration rate by correlating specific GLA production rates with the specific growth rate of the organism using a mixed growth model.

\section{MATERIALS AND METHODS}

\section{Strain and culture medium}

Spirulina platensis strain 21.99 was procured from the
Culture Collection of Algae, University of Goettingen, Germany. The culture was grown in bicarbonate-enriched SOT medium (9). The medium composition on a $\mathrm{g} \cdot \mathrm{L}^{-1}$ basis was as follows: $\mathrm{NaHCO}_{3}, 16.8 ; \mathrm{NaNO}_{3}, 2.5 ; \mathrm{K}_{2} \mathrm{SO}_{4}, 1.0 ; \mathrm{NaCl}, 1.0$; $\mathrm{MgSO}_{4} \cdot 7 \mathrm{H}_{2} \mathrm{O}, \quad 0.2 ; \quad \mathrm{K}_{2} \mathrm{HPO}_{4}, \quad 0.5 ; \quad \mathrm{CaCl}_{2} \cdot 2 \mathrm{H}_{2} \mathrm{O}, \quad 0.04$; $\mathrm{FeSO}_{4} \cdot 7 \mathrm{H}_{2} \mathrm{O}, 0.01$; and EDTA, 0.08. Additionally, the medium contained $1.0 \mathrm{~mL}$ of $\mathrm{A}-5$ trace elemental solution, the elemental composition of which was as follows $\left(\mathrm{g} \cdot \mathrm{L}^{-1}\right): \mathrm{H}_{3} \mathrm{BO}_{3}$, 2.86; $\mathrm{MnCl}_{2} \cdot 4 \mathrm{H}_{2} \mathrm{O}, 1.81 ; \mathrm{ZnSO}_{4} \cdot 7 \mathrm{H}_{2} \mathrm{O}, 0.222 ; \mathrm{Na}_{2} \mathrm{MoO}_{4} \cdot 2 \mathrm{H}_{2} \mathrm{O}$, 0.039; $\mathrm{CuSO}_{4} \cdot 5 \mathrm{H}_{2} \mathrm{O}, 0.079$; and $\mathrm{Co}\left(\mathrm{NO}_{3}\right)_{2} \cdot 6 \mathrm{H}_{2} \mathrm{O}, 0.49$.

\section{Photobioreactor design and operation}

The photobioreactor (Indigenous) was a $0.5-\mathrm{cm}$-thick glass bubble column with an aspect ratio of 1:5 and a radius of $12.5 \mathrm{~cm}$. The operating volume of the reactor was $20 \mathrm{~L}$. Air was sparged through a cross-shaped pipe sparger via thirteen 1$\mathrm{mm}$ holes in each section. The air flow rate was adjusted using a gas flow meter. Continuous illumination was provided by three 18-W daylight fluorescent tubes with an average light intensity of 2000 lx maintained constantly during the operation. All batches were run at room temperature $\left(35^{\circ} \mathrm{C} \pm 1^{\circ} \mathrm{C}\right)$. The $\mathrm{pH}$ of the medium was adjusted to 9.2 before starting the batches. A 10\% inoculum (10-day-old culture grown under similar conditions) was used to inoculate all batches.

To highlight the best operational conditions under which the fatty acid is synthesized, a series of experiments was performed in the bubble column photobioreactor under 3 operational conditions: static, continuous, and periodic aeration. Under static conditions, the reactor was left unagitated during the batch study. In the continuous sparging mode, the compressor operated at a flow rate of $1.2 \mathrm{vvm}$ for 24 h. In the periodic operation mode, the reactor was operated at $1.2 \mathrm{vvm}$ intermittently. Cultivation studies were conducted at 5 different flow rates $(0.2,0.4,0.6,1.2$, and $2.5 \mathrm{vvm})$ in a cycle of $24 \mathrm{~h}$, and aeration was performed periodically $(1 \mathrm{~h}$ on and 1 h off).

\section{Analytical methods}

Dry weight was determined using triplicate samples of the 
cultures $(5 \mathrm{~mL}$ each). Culture samples were washed 1/10 (v/v) with distilled water to dissolve excess salt. The water added to each sample was filtered through Whatman GF/C filters $(1.2 \mu \mathrm{m}$, 47-mm diameter). The filtered cells were dried overnight at $105^{\circ} \mathrm{C}$ until a steady weight was recorded (20). The dissolved oxygen was measured using an Oakton DO6 galvanic probe (Cole-Parmer, USA). The specific growth rate was calculated using a graphical method. The natural logarithm value of the biomass concentration (corresponding to the exponential phase) of the alga was plotted against time, and the slope was used to derive the specific growth rate. The GLA production rate was derived by plotting exponential GLA content ( $\mathrm{mg} \cdot \mathrm{g}^{-1}$ dry cell weight) with time, and the slope was used to estimate the production rate.

The harvested cell culture was subjected to thorough washing with alcohol and hexane to eliminate the remnant slime layer associated with the cells and finally freeze-dried using a lyophilizer. Fatty acids were extracted using a single-step direct transmethylation of freeze-dried algae (7). The fatty acid composition was analyzed using gas chromatography (Thermo Fisher, 8610, Germany). Fatty acids were identified by correlating the retention time with that of standard fatty acids (Sigma \& Co, Germany). Heptadecanoic acid was used as internal standard to quantify individual fatty acids using the internal standard method. TFA content was obtained by considering the sum of quantified individual fatty acids. The column used to identify TFAs was an ethylene glycol succinate-packed column with $10 \% \mathrm{w} / \mathrm{w}$ Chromosorb solid support (12). The column was operated at $180^{\circ} \mathrm{C}$ in the isothermal mode. The mobile phase was inert nitrogen gas fixed at a flow rate of $35 \mathrm{~mL} \cdot \mathrm{m}^{-1}$. Injector and detector temperatures were maintained at $250^{\circ} \mathrm{C}$. A flame-ionizing detector was used in gas chromatography.

\section{Statistical analysis}

All measurements (biomass, GLA, and TFAs) were reported as the mean \pm standard deviation. The experimental data were elaborated by 1 -way analysis of variance; $\mathrm{p}<0.05$ indicated significant differences.

\section{RESULTS AND DISCUSSION}

Fatty acid synthesis in microalgae occurs via 2 pathways: the de novo pathway and the oxygen-dependent pathway. In the former, the production of malonyl $\mathrm{CoA}$ from acetyl $\mathrm{CoA}$ is the rate-limiting step, whereas in the latter, oxygen is rate limiting for the function of $\Delta$-6-desaturase (6).

Figure 1 shows the gradient of dissolved oxygen in the reactor for the different operating conditions. The dissolved oxygen level in the static condition was approximately 2- and 3fold lower than those in the continuous aeration and periodic aeration conditions, respectively. Contrary to the dissolved oxygen profile, a high GLA level of $7.5 \mathrm{mg} \cdot \mathrm{g}^{-1}$ dry biomass was observed with periodic aeration, compared with $6.0 \mathrm{mg} \cdot \mathrm{g}^{-1}$ dry biomass with continuous aeration and $2.3 \mathrm{mg} \cdot \mathrm{g}^{-1}$ dry biomass under a static condition.

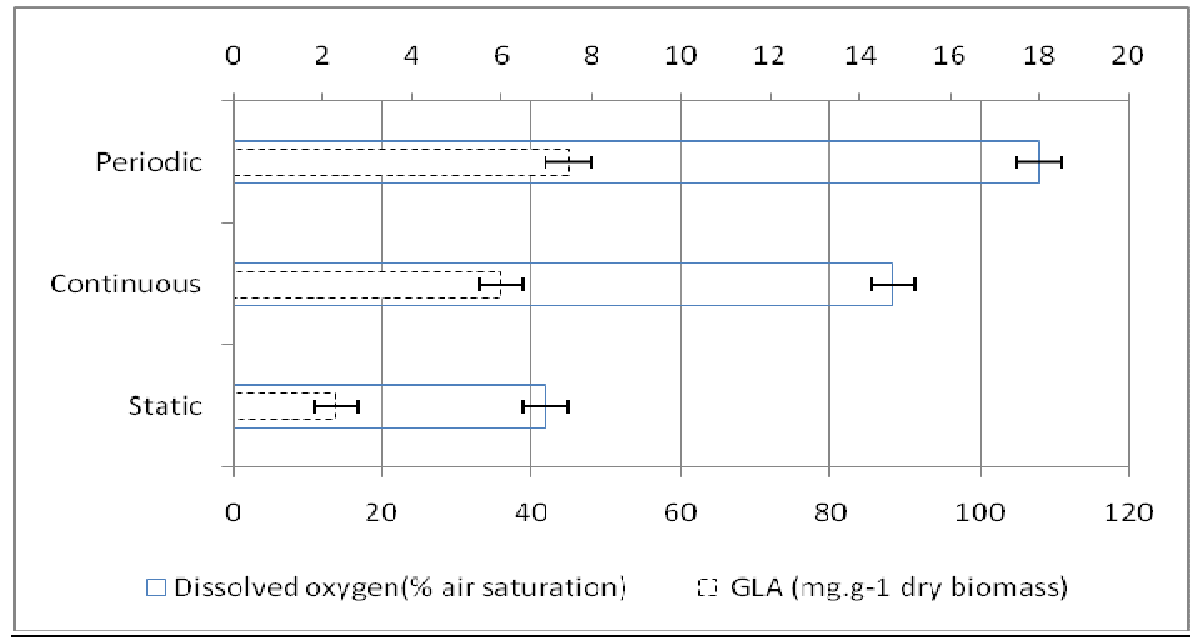

Figure 1. GLA content and percent dissolved oxygen in Spirulina platensis cultured in a bubble column photobioreactor with static, continuous, and periodic sparging. The aeration flow rate of the bioreactor was set at $0.6 \mathrm{vvm}$ during continuous and periodic sparging. 
Higher GLA levels with periodic aeration can be attributed to the exposure of biomass to optimum dissolved oxygen levels, as sufficient time is allowed for oxygen to accumulate during off gas sparging hours. However, in continuous sparging, the dissolved oxygen is well dispersed in the column to maintain dissolved oxygen near saturation levels. Moreover, with the growth of the organism, a gradient of dissolved oxygen exists between the gas-liquid interface and bulk liquid in the medium. Contrary to the continuous aeration condition, poor growth and low dissolved oxygen levels were observed in the static condition; this observation is consistent with the lack of an external oxygen supply and poor mixing in the column.
Figure 2 shows the variation in the biomass productivity and specific growth rate with periodic operation at different aeration rates. An increase in the aeration rate resulted in improvements in both the specific growth rate and biomass productivity. A change in aeration rate from 0.2 to $1.2 \mathrm{vvm}$ resulted in a 2.5 -fold increase in biomass $\left(0.12 \mathrm{~g} \cdot \mathrm{L}^{-1} \cdot \mathrm{day}^{-1}\right)$. A similar trend was also observed with the specific growth rate, which was almost 3-fold higher at a flow rate of $1.2 \mathrm{vvm}$. Any further increase beyond $1.2 \mathrm{vvm}$ had no effect on biomass productivity or the growth rate, indicating $1.2 \mathrm{vvm}$ to be the optimum aeration rate for the growth of algae, and hence, further studies were performed using four flow rates (0.2-1.2 $v v m)$.

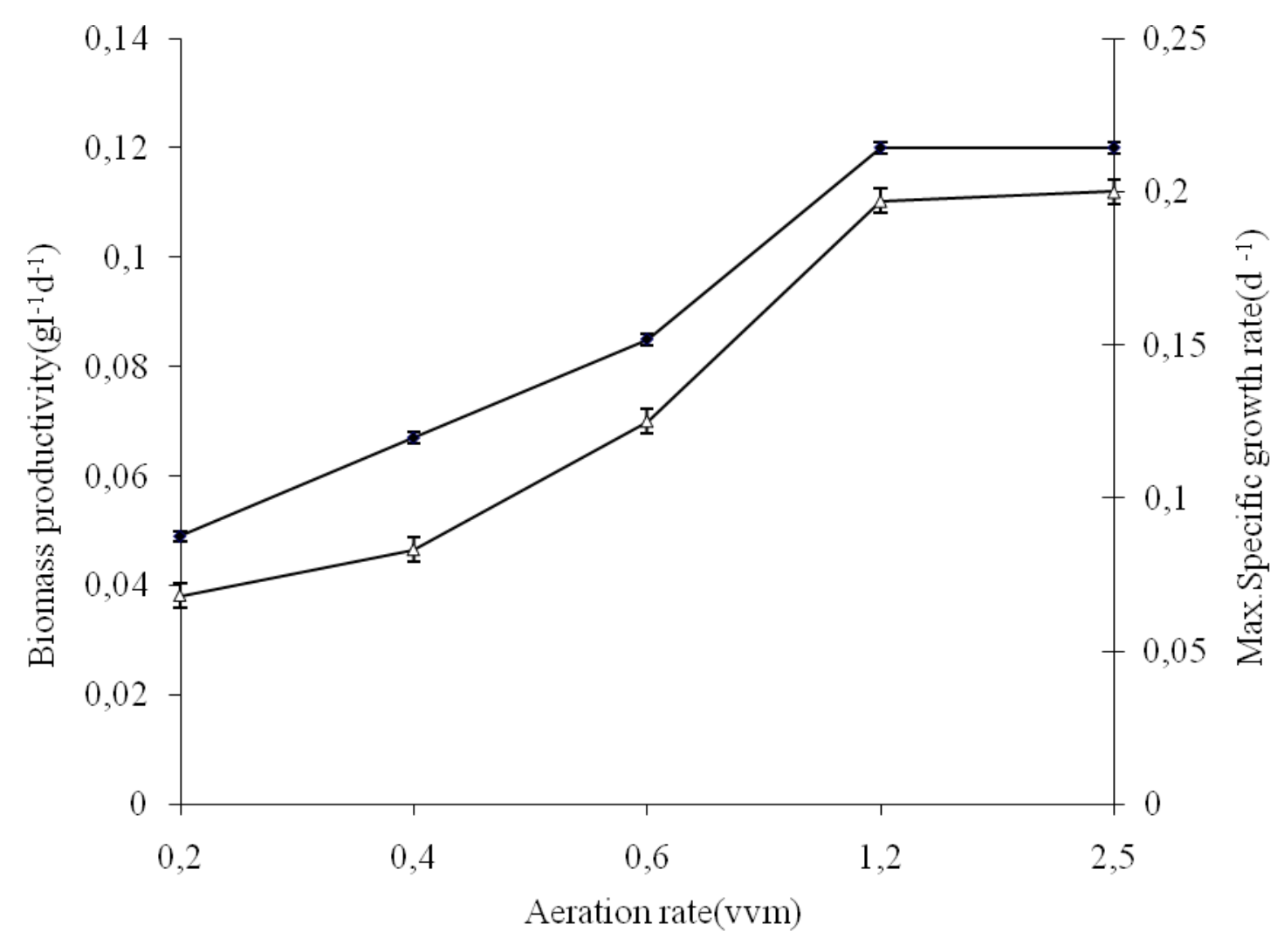

$\rightarrow$ Biomass productivity $\multimap$ Maximum specific growth rate

Figure 2. Effect of aeration rates on algal growth in the bubble column photobioreactor. Biomass productivity and specific growth rates exhibited linear dependence on the aeration rate. 
At the beginning of each batch and at equal intervals of time, algal samples were taken from the reactor and assayed for TFA content. The study of GLA profiles (Figure 3A) revealed that the algae accumulated GLA exponentially followed by a short steady phase and a decline phase in the exponential growth phase of the algae (Figure 3B). Within the tested range of aeration rates, the maximum GLA content increased by $69.64 \%$ (i.e., from 5.6 to $9.5 \mathrm{mg} \cdot \mathrm{g}^{-1}$ dry cell weight). In addition to increasing the GLA content, higher aeration rates also improved the TFA content (from $2.22 \%$ to $4.31 \%$ dry cell biomass), whereas the GLA to TFA ratio remained constant at all flow rates $(22 \%)$, indicating the importance of GLA in maintaining constant membrane fluidity in the algae at the growing temperature (Table 1). The optimum GLA composition obtained in this work correlated with the average GLA content found in TFAs (22\%) in the blue green alga (14). Because all of the experiments were conducted under the same operating conditions excluding the aeration rate, increase in growth and GLA content with changes in the aeration rate are attributed to improved mixing and better oxygen availability. It is well documented that dissolved oxygen is essential for the desaturation of linoleic acid (C18:2) by $\Delta$-6-desaturase in the production of $\operatorname{GLA}(1,23)$. It is significant to attribute this cause to increase in oxygen availability, promoting both growth and the production of GLA by the enzyme. Here, we assume that fatty acid synthesis is only accelerated with both improvements in the mixing of nutrients in the medium, which is required for growth, and partial pressure of oxygen in the air for GLA synthesis. However, restrictions may apply to the synthesis of GLA with improvements in dissolved oxygen concentrations, as there is a possibility of the onset of an oxygen inhibition effect due to saturation of the enzyme ( $\Delta-6$ desaturase) with oxygen at higher aeration rates.

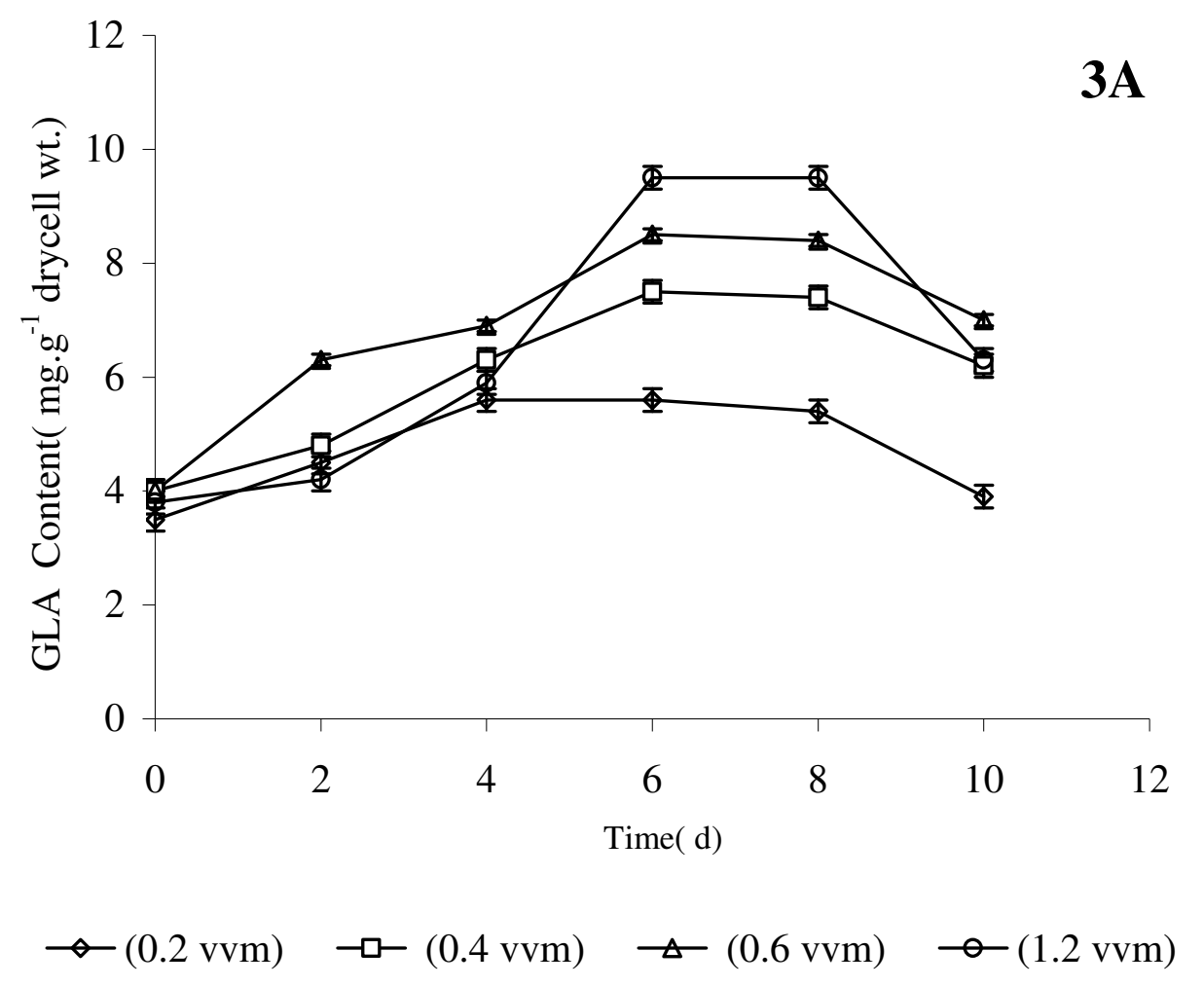




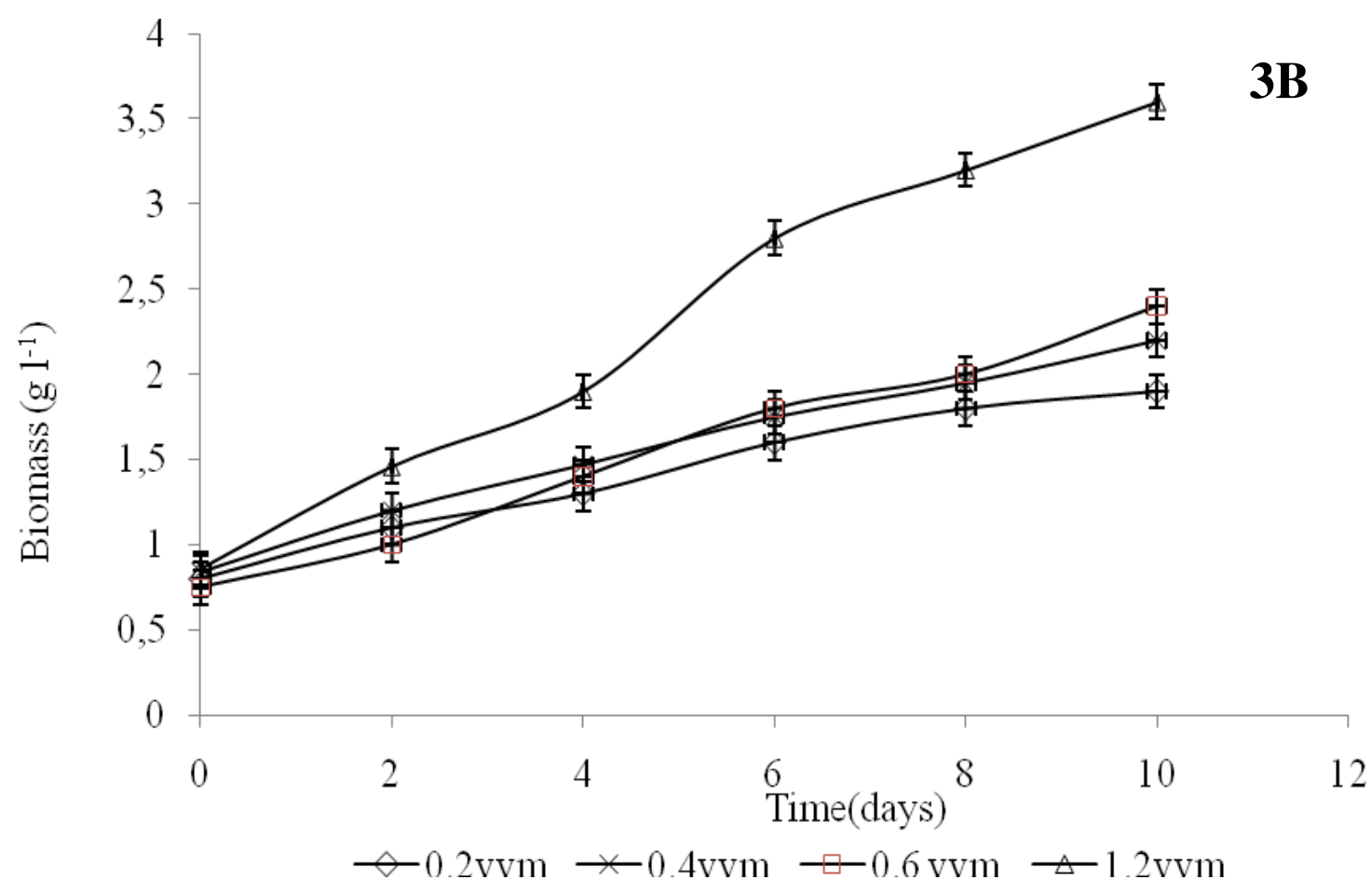

Figure 3. A) GLA content in Spirulina platensis maintained at aeration rates of $0.2,0.4,0.6$, and $1.2 v v m$ during batch growth in the bubble column photobioreactor. B) Biomass variation with time at aeration rates of 0.2, 0.4, 0.6, and $1.2 \mathrm{vvm}$ during batch growth.

Table 1. Total fatty acid (TFA) profile showing the major fatty acid composition of the biomass at aeration rates of $0.2,0.4,0.6$, and $1.2 \mathrm{vvm}$

\begin{tabular}{lcccc}
\hline Fatty acid (\% TFA) & \multicolumn{4}{c}{ Aeration rate $(\boldsymbol{v} \boldsymbol{v m})$} \\
\cline { 2 - 5 } & $\mathbf{0 . 2}$ & $\mathbf{0 . 4}$ & $\mathbf{0 . 6}$ & $\mathbf{1 . 2}$ \\
\hline C16:0 & $50.5 \pm 1.2$ & $48.51 \pm 0.8$ & $51.1 \pm 0.6$ & $50.2 \pm 0.4$ \\
C18:1 & $10.6 \pm 0.3$ & $11.32 \pm 0.2$ & $14.5 \pm 0.3$ & $15.2 \pm 0.2$ \\
C18:2 & $16.7 \pm 0.5$ & $17.27 \pm 0.3$ & $11.4 \pm 0.1$ & $12.2 \pm 0.5$ \\
C18:3 & $22.06 \pm 0.4$ & $21.99 \pm 0.3$ & $22.3 \pm 0.2$ & $22.0 \pm 0.3$ \\
\hline
\end{tabular}

Although the direct influence of aeration on GLA production cannot be clarified quantitatively, useful information can still be obtained from the growth and product formation data. The dependence of fatty acid production on aeration can be best explained by associating the rate of fatty acid production with that of algal growth, which in turn depends on aeration. The specific growth rate and biomass productivity are linearly dependent on the aeration rate up to $1.2 \mathrm{vvm}$ (Figure 2). Thus, a correlation between the specific growth rate and GLA would provide an indirect relationship between GLA content and the aeration rate. Many models cited in the literature are available to explain product formation with respect to growth of the organism. Among them, the Luedeking and Piret model (11) predicts growth-associated and non- 
growth-associated product formation. The basic equation includes both growth-related $(\alpha)$ and non-growth-related $(\beta)$ variable constants.

According to Luedeking and Piret (1959),

$$
d G / d t=\alpha d C / d t+\beta C
$$

where $G$ is gamma-linolenic acid, $C$ is cell density, $t$ is time, and $\alpha$ and $\beta$ are constants.

In the above equation, if $\alpha=0$, then the product is nongrowth-associated, whereas if $\beta=0$, then the product is growth-associated.

$$
\begin{aligned}
& \text { Additionally, because } d C / d t=\mu C \\
& \qquad d G / d t=\alpha \mu C+\beta C, \\
& \text { then },
\end{aligned}
$$

where $\mu$ is the specific growth rate.

In addition, $d G / d t=q_{G} C$ where $q_{G}$ is the specific rate of product formation.

Then,

$$
q_{G}=\alpha \mu+\beta
$$

Plotting $q_{G}$ vs. $\mu$ (Figure 4) illustrates the relationship between the specific production rate and the specific growth rate. The linear relationship obtained from the plot was

$$
q_{G}=4.44 \mu+0.18
$$

Because the $\alpha$ parameter (4.44) dominates the $\beta$ parameter (0.18), the product (GLA) can be regarded as growthassociated. However, it was observed that the algal GLA content decreased beyond day 8 of cultivation (Figure 3A), whereas the cell concentration continued to increase beyond day 10 (Figure 3B). This indicates that within a certain period of cultivation time, the increase in GLA content strongly depends on algal growth, which is influenced by the aeration rate.

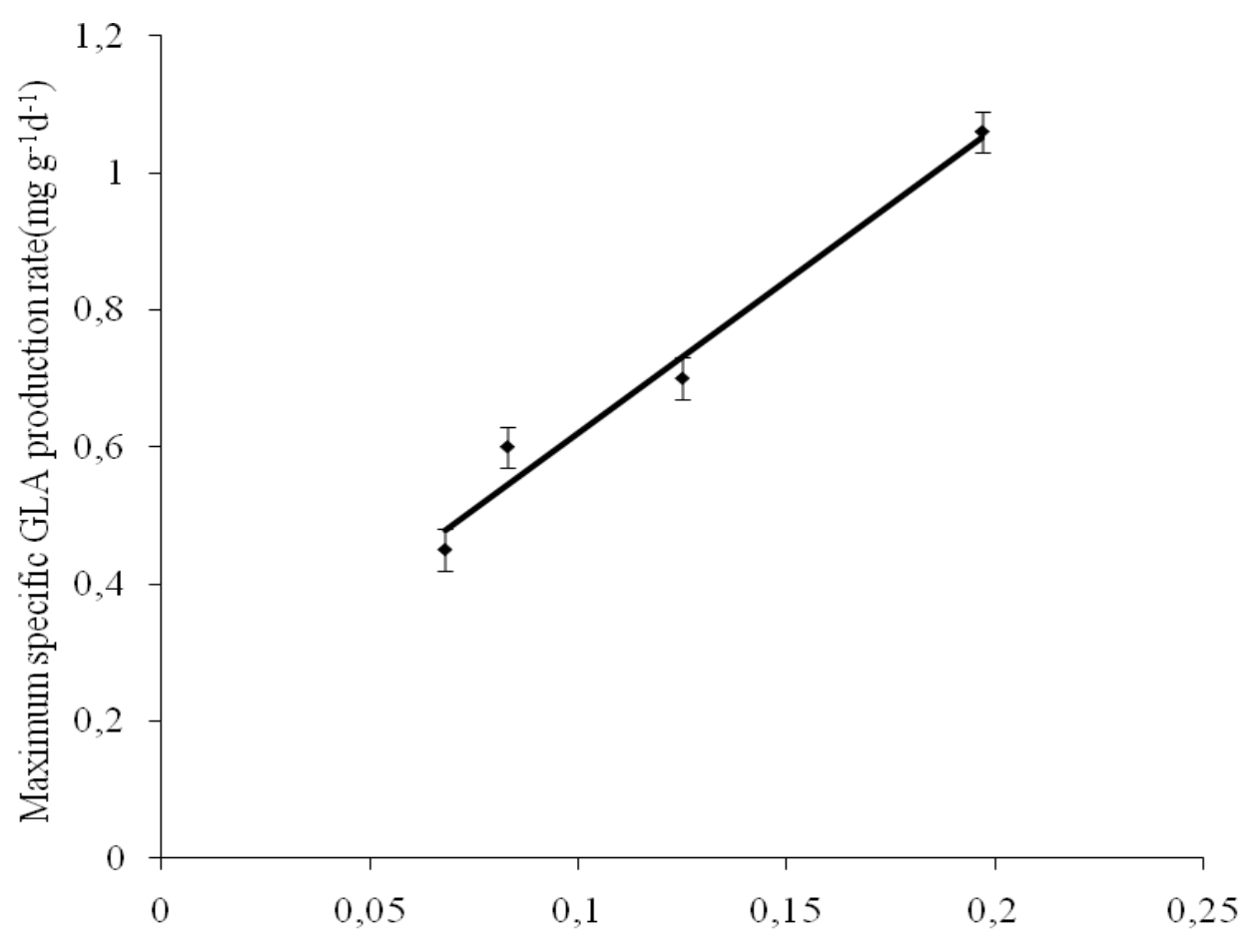

Maximum specific growth rate $\left(\mathrm{d}^{-1}\right)$

Figure 4. Comparison between the maximum specific GLA production rate and maximum specific growth rates of Spirulina platensis calculated on the peak day of GLA production (day 6). 
Algal cultivation with bicarbonate-enriched medium under static, continuous, and periodic operation of air exhibited periodic sparging suitable for GLA production. The aeration rate was optimized for algal growth for further studies of its effects on GLA production. Increase in the aeration rate with periodic aeration enhanced the overall reactor performance, i.e., specific growth rate, GLA content, and TFA content. Despite changes in the aeration rate, the GLA to TFA ratio remained constant $(22 \%)$, indicating the level of GLA required among TFAs to maintain the membrane fluidity of the algae at the growing temperature. The application of the Luedeking and Piret model demonstrated the dependency of GLA production on aeration. It was found that GLA production depends on algal growth (biomass), which is further influenced by aeration. In the present work, kinetic studies of GLA production illustrated that the commercial exploitation of Spirulina can be achieved for GLA production by shortening the harvesting time (between day 6 and 8) at optimum aeration rates. To understand the role of dissolved oxygen on GLA content, studies on the enzyme regulatory mechanism is necessary. This would allow us to better determine the contribution of oxygen to the synthesis of high-value products such as GLA.

\section{ACKNOWLEDGEMENTS}

The authors would like to thank the Department of Science and Technology, Government of India, for supporting this work.

\section{REFERENCES}

1. Ahmed, S.U.; Singh, S.K.; Pandey, A.; Kanjilal, S.; Prasad, R.B.N. (2009). Application of response surface method for studying the role of dissolved oxygen and agitation speed on gamma linolenic acid production. Appl. Biochem. Biotechnol. 152(1), 108-116.

2. Alvala, D.; Mello, P.C.; Wagener, K. (1997). The relevance of $\mathrm{CO}_{2}$ partial pressure of sodium bicarbonate solutions for the mass cultivation of the microalga Spirulina. J. Brazil Chem. Soc. 8(5), 447-450.

3. Belarbi, E.H.; Molina, E.; Chisti, Y. (2000). A process for high yield and scalable recovery of high purity eicosapentaenoic acid esters from microalgae and fish oil. Enzyme Microb. Technol. 26(7), 516-529.

4. Borowitzka, M.A. (1999). Pharmaceuticals and agrochemicals from microalgae. In: Cohen Z, editor. Chemicals from Microalgae. Taylor \& Francis, London, UK, p. 313-352.

5. Bugnariu, S. (1996). Beneficial effect of gamma-linolenic acid-enriched diet therapeutic properties of Oenothera biennis oil (evening primrose oil). Farmacia 44, 3-4.

6. Certik, M.; Shimizu, S. (1999). Biosynthesis and regulation of microbial polyunsaturated fatty acid production. J. Biosci. Bioeng. 87(1), 1-14.

7. Cohen, Z.; Reungjitchachawali, M.; Siangdung, W.; Tanticharoen, M. (1993). Production and partial purification of $\gamma$-Linolenic acid and some pigments from Spirulina platensis. J. Appl. Phycol. 5(1), 109-115.

8. Cohen, Z.; Vonshak, A. (1990). Fatty acid composition of Spirulina and Spirulina-like cyanobacteria in relation to their chemotaxonomy. $J$. Photochem. 30(1), 205-206.

9. Hirano, M.; Mori, H.; Miura, Y.; Matsunaga, N.; Matsunaga, T. (1990). $\gamma$-Linolenic acid production by microalgae. Appl. Microbiol. Biotechnol. 24-25, 183-191.

10. Kshirsagar, H.H.; Revankar, M.S.; Kamat, M.Y.; Lele, S.S. (2002). Cultivation of Spirulina in gas induced photobioreactor and isolation of phycobiliproteins. Indian J. Biotechnol. 1, 255-262.

11. Leudeking, R.; Piret, E.L. (1959). A kinetic study of the lactic acid fermentation. J. Biochem. Microbiol. 1(4), 393-412.

12. Mahajan, G.; Kamat, M. (1999). $\gamma$-Linolenic acid production from Spirulina platensis. Appl Microbiol. Biotechnol. 43(3), 466-469.

13. Molina, E.; Fernandez, J.; Acien, F.G.; Christi, Y. (2001). Tubular photobioreactor design for algal cultures. J. Biotechnol. 92(2), 113-131.

14. Qiang, H.; Zheng, H.; Cohen, Z.; Richmond, A. (1997). Enhancement of eicosapentaenoic acid (EPA) and $\gamma$-linolenic acid (GLA) production by manipulating algal density of outdoor cultures of Monodus subterraneus (Eustigmatophyta) and Spirulina platensis (Cyanobacteria). Eur. J. Phycol. 32(6), 81-86.

15. Rodriguez-Maroto, J.M.; Carlos, J.; Aguilera, A.; Niell, F.X. (2005). Air bubbling results in carbon loss during microalgal cultivation in bicarbonate-enriched media: experimental data and process modeling. Aquacult. Eng. 32(3-4), 493-508.

16. Ronda, S.R.; Lele, S.S. (2008). Culture conditions stimulating high gamma linolenic acid accumulation by Spirulina platensis. Braz. J. Microbiol. 39(4), 693-697.

17. Sanchez, M.A.; Garcia, F.C.; Contreras, A.; Molina, E.G.; Chisti, Y. (2000). Bubble column and airlift photobioreactors for algal cultures. Am. Inst. Chem. Eng. J. 46(9), 1872-1887.

18. Specktorova, L.; LeRoy.; R Creswell, R.; Vaughan, D. (1997). Closed tubular cultivations: an innovative system for commercial culture of microalgae. World Aquac. 28, 39-43.

19. Tredici, M.R.; Zittelli, G.C. (1998). Efficiency of sunlight utilization: 
Tubular versus Flat photobioreactors. J Biotechnol. Bioeng. 57(2), 187197.

20. Vonshak, A. (1997). Spirulina platensis (Arthrospira): physiology, cell biology and biotechnology. Taylor \& Francis Publishers, London

21. Worm, M.; Henz, B.M. (2000). Novel unconventional therapeutic approaches to atopic eczema. Dermatology 201(3), 191-195.
22. Zurier, R.B.; Rossetti, R.G.; Jacobson, E.W.; DeMarco, D.M.; Liu, N.Y.; Temming, J.E.; White, B.M.; Laposata, M. (1996). Gammalinolenic acid treatment of rheumatoid arthritis. A randomized, placebo-controlled trial. Arthritis Rheum. 39(11), 1808-1817.

23. Zvi Cohen.; Yair, M.; Heimer, A. (1990). Novel Mode of Action of Norflurazone. Plant Physiol. 93, 347-349.

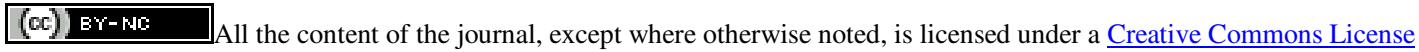

